\title{
L-selectin and SDF-1 enhance the migration of mouse and human cardiac mesoangioblasts
}

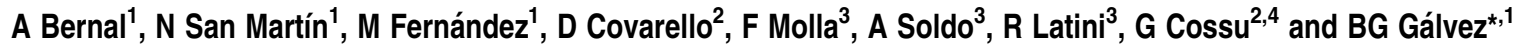

Efficient delivery of stem cells to heart regions is still a major problem for cell therapy. Here, we report experiments aimed to improve migration of mouse and human cardiac mesoangioblasts to the damaged heart. Cardiac mesoangioblasts were induced to transmigrate through the endothelium by factors released by cardiomyocytes or cytokines, among which stromal-derived factor 1 (SDF-1) was the most potent. Cardiac mesoangioblasts were also delivered into the left ventricular (LV) chamber of mice after coronary artery ligation (CAL), and their in vivo homing to the damaged heart was found to be quite modest. Pretreatment of cardiac mesoangioblasts with SDF-1 or transient expression of L-selectin induced a two- to three-fold increase in their transmigration and homing to the damaged heart. Therefore, combined pretreatment with SDF-1 and L-selectin generated modified cardiac mesoangioblasts, $50 \%$ of which, after injection into the LV chamber of mice early after CAL, home directly to the damaged free wall of the heart. Finally, modified mouse cardiac mesoangioblasts, injected into the LV chamber regenerate a larger surface of the ventricle in long-term experiments in comparison with their control counterparts. This study defines the requirements for efficient homing of cardiac mesoangioblasts to the damaged heart and offers a new potent tool to optimize efficiency of future cell therapy protocols for cardiovascular diseases.

Cell Death and Differentiation (2012) 19, 345-355; doi:10.1038/cdd.2011.110; published online 26 August 2011

Homing is the process by which cells reach a specific target tissue through the general circulation. This mechanism has been studied extensively both in vitro and in vivo with different cell types, such as leukocytes or hematopoietic stem cells ${ }^{1,2}$ and is believed to rely on adhesion molecules and cytokine receptors, consisting of a rolling interaction with the endothelium, followed by firm adhesion and transmigration into the surrounding tissue. ${ }^{3-5}$

The repertoire of cytokine expression by the target tissue and expression of the relative receptors on endothelial cells influence the efficiency of homing. During inflammation that follows injury or disease, cytokines are involved not only in the recruitment of leukocytes but also in that of circulating stem cells. ${ }^{6,7}$ In fact, stromal-derived factor (SDF-1) and VEGF favor the arrest of progenitors on the vascular endothelium, whereas interleukin-8 (IL-8) promotes stem cell mobilization from the bone marrow. ${ }^{8-10}$ HMGB1 and tumor necrosis factoralpha $(T N F-\alpha)$ have also been described as signals that induce migration of different mesenchymal cells. ${ }^{11-13}$ Although these mechanisms have been elucidated to a large extent for leukocytes, far less is known for the new types of recently described stem cells and this limits the possibility of their efficient delivery to target tissues.

Cardiac mesoangioblasts were recently characterized as a population of mesenchymal stem cells that differentiate efficiently into cardiomyocytes (CMCs). ${ }^{14,15}$ They have been previously shown to restore to a significant extent heart structure and function in a mouse model of coronary artery ligation $(\mathrm{CAL}){ }^{14}$ One main reason for the partial effect of cardiac mesoangioblasts in this model is likely to be ascribed to the limited homing of these cells to the heart. Cardiac mesoangioblast extravasation must be directed by selective precursor-endothelial cell recognition.

To increase the efficiency of cardiac repair by cardiac mesoangioblasts, it would be essential to increase their homing and survival in the heart, with the additional benefit of reducing unspecific trapping in the capillary filters of the body. To this aim, we investigated the role of some adhesion molecules and cytokines in the transmigration of cardiac mesoangioblasts in vitro through the endothelial monolayer and in vivo, their migration in the infarct area of the heart in mice which underwent permanent CAL. ${ }^{15}$ Here, we report that expression of L-selectin and exposure of cells to SDF-1 improve up to five-fold the homing of cardiac mesoangioblasts to the infarcted free wall of the heart. We also show that this treatment improves the recovery of the left ventricular (LV) wall motion after infarction and the quantity of regenerated myocardium. These results elucidate the homing mechanism of cardiac mesoangioblasts and open a new opportunity for improving efficacy of cell therapy in cardiovascular diseases.

${ }^{1}$ Department of Regenerative Cardiology, Centro Nacional de Investigaciones Cardiovasculares, Madrid, Spain; ${ }^{2}$ Division of Regenerative Medicine, San Raffaele Scientific Institute, Milan, Italy; ${ }^{3}$ Department of Cardiovascular Research, Mario Negri Institute, Milan, Italy and ${ }^{4}$ Department of Biology, University of Milan, Milan, Italy *Corresponding author: BG Gálvez, Department of Regenerative Cardiology, Centro Nacional de Investigaciones Cardiovasculares, C/ Melchor Fernandez Almagro, 3., 28029-Madrid, Spain. Tel: + 34914531200 (3317); Fax: + 34 914531240; E-mail: bgonzalez@ cnic.es

Keywords: migration; regeneration; homing; stem cells; cytokines

Abbreviations: $A b$, antibody; CAL, coronary artery ligation; CMC, cardiomyocyte; EGFP, enhanced green fluorescence protein; FGF, fibroblast growth factor; siRNA, small-interference RNA; MMP, metaloproteinase; SDF-1, stromal-derived factor-1; TNF- $\alpha$, tumor necrosis factor-alpha

Received 12.5.11; revised 17.6.11; accepted 13.7.11; Edited by P Salomoni; published online 26.8.11 


\section{Results}

Neonatal CMCs and SDF-1 favor cardiac mesoangioblast transmigration in vitro. Cardiac mesoangioblasts were starved for $12 \mathrm{~h}$ in the absence of serum and then subjected to transmigration assays using Transwell chambers. We created an artificial environment in which cardiac mesoangioblasts would face a TNF- $\alpha$-activated endothelium separating them from neonatal CMCs or specific cytokines. As shown in Figure 1a, cardiac mesoangioblasts were unable to cross endothelium-coated filters in the absence of stimuli. However, neonatal CMCs in culture induced active migration (sixfold above control) of cardiac mesoangioblasts but not of primary fibroblasts. This effect was slightly stronger than that induced by fibroblast growth factor (FGF), used as a positive control. We also performed a chemotaxis assay through endothelium-coated filters with a panel of cytokines. As shown in Figure 1a, the presence of SDF-1 in the lower chamber caused an eight-fold increase in mesoangioblast migration, a significantly more robust effect than that elicited by FGF, MCP-1 or TNF- $\alpha$, previously described as enhancers of mesoangioblast migration. $^{13}$ Interestingly, when an antagonist for CXCR4 (AMD3100) was used, CMC-induced mesoangioblast migration was abolished by $40 \%$ demonstrating a direct correlation between SDF-1-induced signaling and migration of cardiac mesoangioblasts. Besides, as shown in Figure 1b, SDF-1, MCP-1 and IL-8 were detected at higher levels in the supernatant from CMCs than in fibroblasts. These data suggest that differentiated CMCs may secrete cytokines such as SDF-1 or MCP-1 that favor cardiac mesoangioblast transmigration by either a chemotactic effect and/or by modifying the endothelium barrier.

a

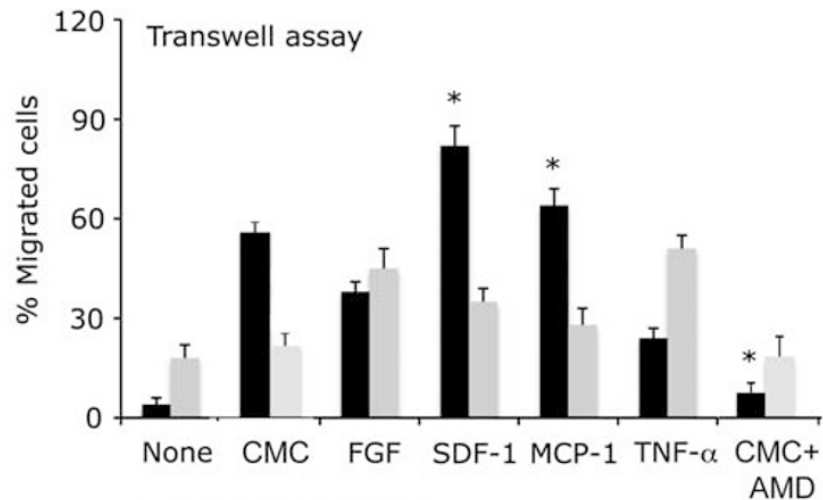

- Cardiac precursors

AMD

Fibroblasts
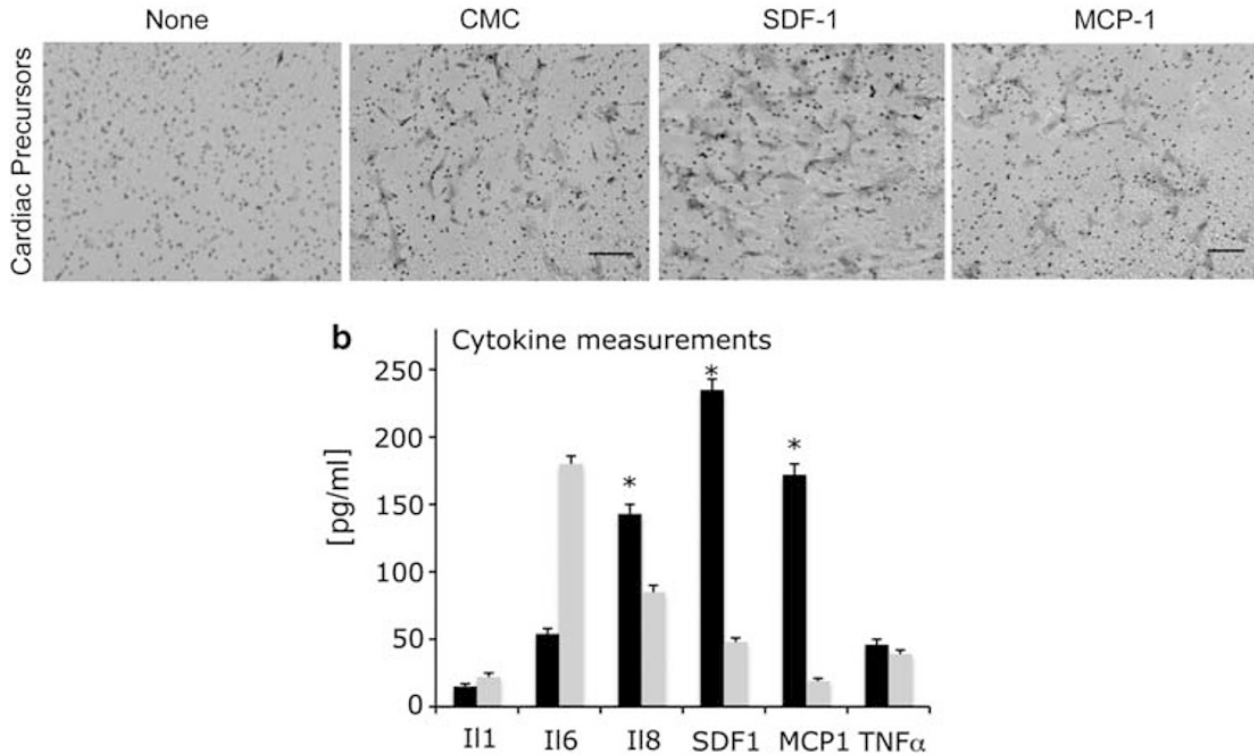

- Cardiomyocytes

Fibroblasts

Figure 1 Induction of cardiac mesoangioblast transmigration by cytokines in vitro. (a) Mouse cardiac mesoangioblasts or fibroblasts were plated on endothelium-coated Transwell filters and induced to migrate for $6 \mathrm{~h}$ in presence of cardiomyocytes (CMCs) or different cytokines ( $30 \mathrm{ng} / \mathrm{ml} \mathrm{FGF;} 50 \mathrm{ng} / \mathrm{ml} \mathrm{SDF}-1 ; 80 \mathrm{ng} / \mathrm{ml} \mathrm{MCP-1;30} \mathrm{ng/ml} \mathrm{TNF-} \alpha$; $10 \mathrm{ng} / \mathrm{ml}$ AMD3100, CXCR4 antagonist). A representative out of five independent experiments run in duplicate is shown (left; ${ }^{*} \alpha<0.02$ ). A representative image of the transmigrated cardiac mesoangioblasts in the presence of CMCs, SDF-1 or MCP-1 is also shown. Bar, $30 \mu \mathrm{m}$. (b) Supernatants from fibroblasts or CMCs were collected after 4 days, and different cytokines were detected using the mouse multi-cytokine detection system. One out of three experiments is shown $\left({ }^{*} \alpha<0.025\right)$ 

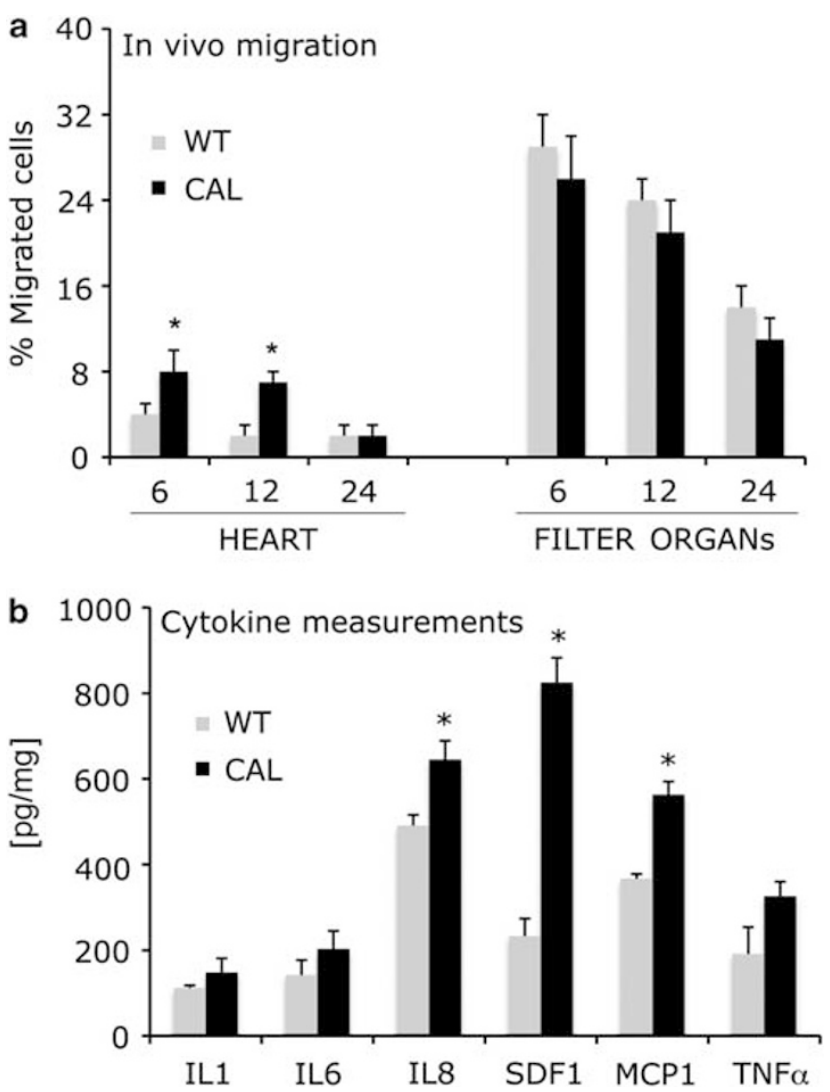

Figure 2 Homing of cardiac mesoangioblasts in vivo. (a) Cardiac mesoangioblasts were injected into the LV chamber of mice subjected to coronary artery ligation (CAL) or control mice. After 6,12 or $24 \mathrm{~h}$, the heart and filter organs were collected and the number of migrated cells was calculated by real-time PCR for GFP. A mean of three independent experiments run in triplicate is shown $\left({ }^{*} \alpha<0.03\right)$. (b) Myocardium free walls of the hearts from 2-month-old WT or CAL mice were homogenized and analyzed for cytokine expression using the mouse multi-cytokine detection system. Concentrations have been normalized to protein load. One out of three experiments is shown $\left({ }^{*} \alpha<0.025\right)$

Cardiac mesoangioblasts and homing in vivo to the heart. A property of stem cells is their ability to migrate through different routes. Clinical trials for cardiovascular diseases have had modest success, possibly because of inefficient delivery of donor cells to the heart. To assess the ability of cardiac mesoangioblasts to migrate in vivo, we injected $5 \times 10^{5}$ GFP-labeled cardiac mesoangioblasts into the LV chamber of wild-type (WT) C57 mice and mice that had undergone CAL few hours earlier. Mice were killed 6, 12 or $24 \mathrm{~h}$ after injection; the heart (free wall) and filter organs (such as the liver, spleen and lungs) were collected and RNA was extracted. The percentage of migrated cardiac mesoangioblasts in each recipient organ was calculated by real-time PCR for GFP expression as a percentage of the value corresponding to the total of injected cells. As shown in Figure $2 \mathrm{a}$, cardiac mesoangioblasts that migrated to the heart in control mice (gray columns), were $\sim 5 \%$ of injected cells, whereas most of the injected cells were retained in the different filter organs, without reaching the heart. Interestingly, the presence of an infarct in the CAL model (black columns) induced an enhanced migration (twofold) of cardiac mesoangioblasts to the infarct zone, compared with their control counterparts. Most of the injected cells were again retained in the filter organs, but this time $\sim 10 \%$ of injected cells could reach the damaged area of the heart. Similar results were obtained when real-time PCR was performed for the $y$ chromosome as double control (Supplementary Figure S1a). We set our time of analysis at $6 \mathrm{~h}$ after injection for the following in vivo experiments, as we observed that the number of injected cells remains constant for the first $12 \mathrm{~h}$ after the injection (Figure 2a). As shown in Figure $2 \mathrm{~b}$, the higher concentration of SDF-1, II-8 and MCP-1 in the free wall of hearts from CAL mice in comparison with control mice could explain the preferential homing of cardiac mesoangioblasts.

Pretreatment of cardiac mesoangioblasts with SDF-1 increases their migration. In an attempt to increase the number of cardiac mesoangioblasts that home specifically to the infarcted zone, we followed two approaches. First, we pretreated cardiac mesoangioblasts with several cytokines for $12 \mathrm{~h}$ before challenging them in the Transwell assay. The results, shown in Figure $3 a$, indicated that pretreatment with SDF-1 increased cardiac mesoangioblast migration to the same extent observed when the same cytokine was present in the lower chamber. This pretreatment did not modify the surface selection markers nor the differentiation profile of the cardiac mesoangioblasts described previously. ${ }^{14}$ Therefore, SDF-1-pretreated GFP-cardiac mesoangioblasts were injected into the LV chamber of control WT or CAL mice, and after $6 \mathrm{~h}$, the hearts and filter organs were collected and analyzed by real-time PCR for the presence of GFP. As shown in Figure 3b, SDF-1 increased about threefold the homing of cardiac mesoangioblasts to the injured area of the heart in mice that underwent CAL. Consistently, the number of cardiac mesoangioblasts detected in the filter organs was reduced to almost half of control.

We then attempted to identify the surface molecules responsible for the changes observed in cardiac mesoangioblast migration after pretreatment with SDF-1. Therefore, we performed a microarray analysis for 125 genes including surface receptors (Figure $3 c$ ). Five genes were significantly modified after pretreatment of cardiac mesoangioblasts with SDF-1: MMP-2, MMP-9, CD44, caveolin-1 and Itg $\alpha \mathrm{v}$. To verify the role of those molecules in cardiac mesoangioblast transmigration, we repeated the Transwell assay in the presence of antibodies (Abs) against CD44 or $\alpha \mathrm{V}$-integrin, metaloproteinase (MMP) inhibitor GM1489 or caveolin-1 (cav-1) small-interference RNA (siRNA) (Figure 3d). As shown in Figure 3d, Abs against CD44 or cav-1 siRNA significantly inhibited SDF-1-induced in vitro transmigration. Blocking MMPs' activity with GM1489 slightly inhibited transmigration induced by SDF-1.

Taken together, these data suggest that SDF-1 increases cardiac mesoangioblast transmigration in vitro and homing to the heart in vivo by increasing expression of several molecules among which CD44 seems to be preferentially expressed.

L-selectin is necessary for efficient cardiac mesoangioblast transmigration and homing to the heart. As revealed by microarray analysis (Tagliafico $E$ 
a
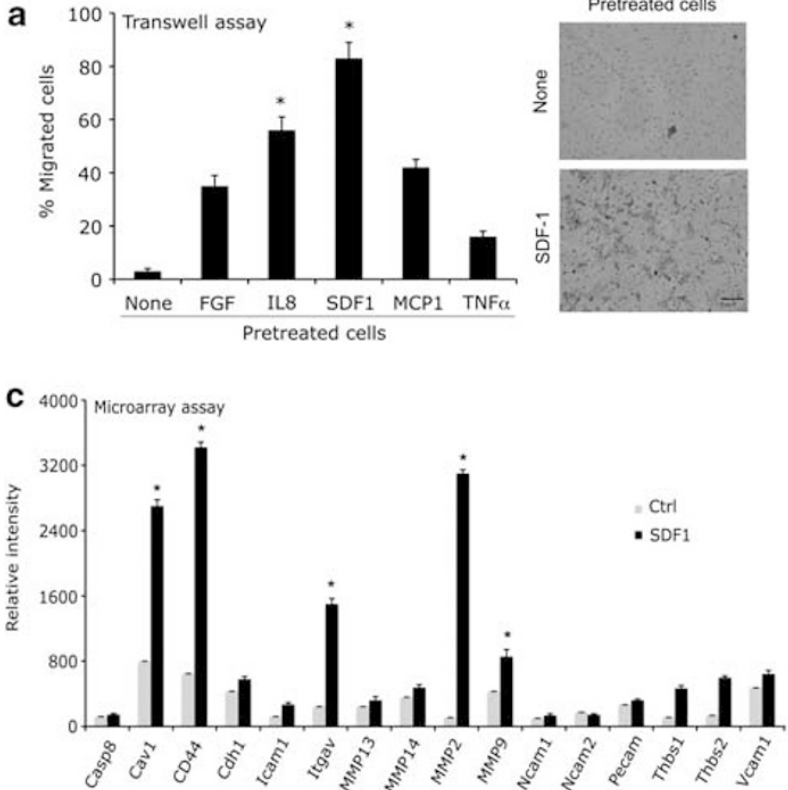

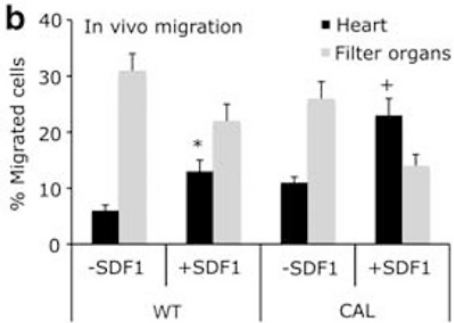

d

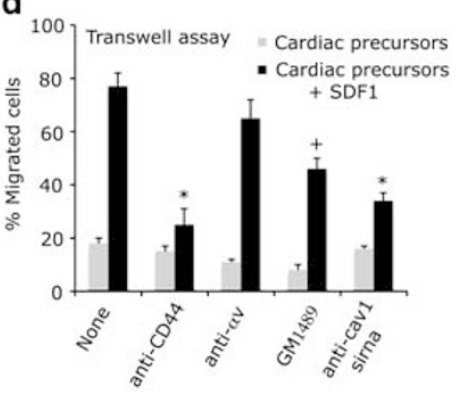

Figure 3 SDF-1 improves cardiac mesoangioblast migration through CD44, MMPs and cav-1. (a) Cardiac mesoangioblasts pretreated with different cytokines $(50 \mathrm{ng} / \mathrm{ml}$

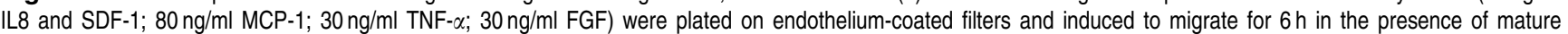
cardiomyocytes in the lower chamber. FGF was used as a positive control. One representative out of five independent experiments run in duplicate is shown (left; ${ }^{*} \alpha<0.01$ ). A representative image of the transmigrated SDF-1-pretreated cardiac mesoangioblasts is also shown (right). Bar, $30 \mu \mathrm{m}$. (b) GFP-cardiac mesoangioblasts pretreated with $50 \mathrm{ng} / \mathrm{ml} \mathrm{SDF}-1$ were injected into the LV chamber of control or CAL mice, and after $6 \mathrm{~h}$, the heart and filter organs were analyzed by real-time PCR for the presence of migrated cells $\left({ }^{*} \alpha<0.03,{ }^{+} \alpha<0.02\right)$. (c) A gene array containing oligos corresponding to different mouse adhesion molecules was hybridized with cDNAs probes retrotranscribed from RNA of cardiac mesoangioblasts pretreated with SDF-1. Relative RNA levels of selected mRNAs normalized to $\beta$-actin expression are shown. Data presented are the mean of two independent experiments $\left({ }^{*} \alpha<0.01\right)$. (d) SDF-1-pretreated cardiac mesoangioblasts were incubated with the MMP inhibitor, GM1489 or siRNA cav-1 inhibitor or with Abs against CD44 and $\alpha \mathrm{v}$ integrin and induced to migrate for $6 \mathrm{~h}$ through endothelium-coated filters and in the presence of mature cardiomyocytes in the lower chamber. A representative out of three independent experiments run in duplicate is shown $\left({ }^{*} \alpha<0.02,{ }^{+} \alpha<0.005\right)$

and Cossu G, unpublished data), cardiac mesoangioblasts do not express some of the key molecules that control rolling and extravasation of leukocytes, such as L-selectin or $\beta 2$ integrin. ${ }^{1,5}$ Therefore, we transduced mesoangioblasts with vectors expressing these molecules and also enhanced green fluorescence protein (EGFP) that were used previously by our group. ${ }^{13}$ These constructs did not modify the surface expression markers nor the differentiation profile of the cardiac mesoangioblasts described previously. ${ }^{14}$ To assess whether the expression of these molecules could improve cardiac mesoangioblast migration, cells transfected with one or both constructs were subjected to transmigration in vitro assay through the activated endothelium. As shown in Figure $4 \mathrm{a}$, L-selectin expression increased by six- to eightfold cardiac mesoangioblast transmigration, whereas $\beta 2$ integrin expression increased only by twofold their migration. Co-transfection of both constructs had no statistically significant cumulative effect (Figure 4a). When L-selectin-expressing cardiac mesoangioblasts were injected into the LV chamber of mice early after CAL, the number of cells that reached the free wall of the heart increased approximately twofold in different mice (Figure 4b). Consistent with in vitro results, we did not observe any cumulative effect with co-transfection of L-selectin and $\beta 2$ integrin. Therefore, expression of L-selectin by cardiac mesoangioblasts improves their migration and homing possibly by favoring their interaction with ligands expressed on the endothelium surface.
Pretreatment with SDF-1 of L-selectin-expressing cardiac mesoangioblasts increased by fivefold their homing to the heart. We then tested the possibility of additive or synergistic effects of the two approaches shown above. Cardiac mesoangioblasts previously transfected with L-selectin constructs were pretreated for $12 \mathrm{~h}$ with SDF-1 and then subjected to transmigration assay through the endothelium. As shown in Figure 4c, the combination of SDF-1 pretreatment and L-selectin expression seemed to have a slightly synergistic effect and improved cardiac mesoangioblast transmigration in vitro 15 -fold more efficiently than control. Therefore, these cells were injected into the LV chamber of infarcted mice. As shown in Figure $4 \mathrm{~d}$, results showed that almost $50 \%$ of injected cells reached the injured myocardium of the heart after $6 \mathrm{~h}$, with a concomitant reduction in the number of cardiac mesoangioblasts detected in filter organs (Figure 4d). Again, SDF-1 pretreatment and L-selectin expression demonstrated a clear synergistic effect in in vivo experiments. Similar results were obtained when real-time PCR was performed for the y chromosome as double control (Supplementary Figure S1b).

Myocardium regeneration after long-term treatment with modified cardiac mesoangioblasts. Finally, to assess the ability of modified cardiac mesoangioblasts not only to migrate in vivo to the heart but also to regenerate the myocardium, we injected $5 \times 10^{5}$ GFP-labeled control or 

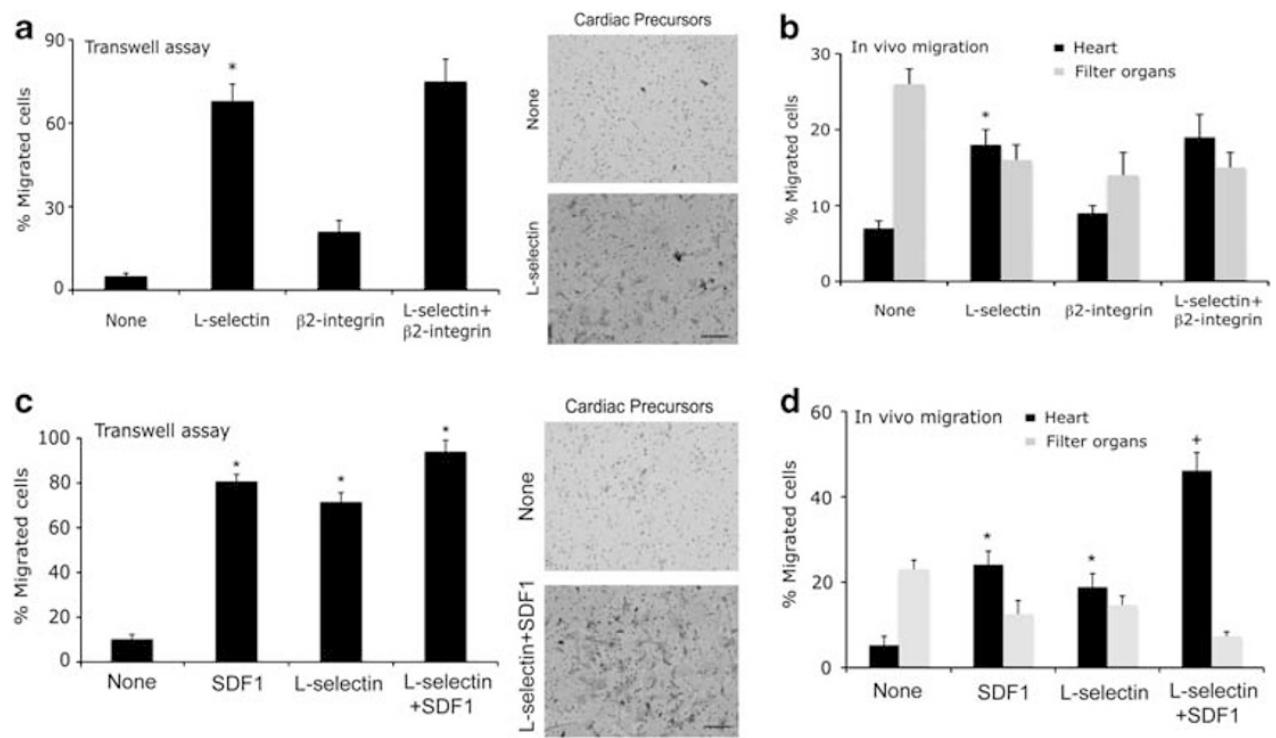

Figure 4 L-selectin increases cardiac mesoangioblast migration and has synergistic effects with SDF-1 pretreatment. (a) Cardiac mesoangioblasts transfected with one or two vectors expressing different surface molecules and EGFP were plated on endothelium-coated filters and induced to migrate for $6 \mathrm{~h}$ in the presence of mature cardiomyocytes in the lower chamber. A representative out of five independent experiments run in duplicate is shown (left panel) (left; ${ }^{*} \alpha<0.01$ ). A representative image of the transmigrated cardiac mesoangioblasts transfected with L-selectin is also shown (right). Bar, $30 \mu \mathrm{m}$. (b) Cardiac mesoangioblasts transfected with vectors expressing L-selectin and/or $\beta 2$-integrin and GFP were injected into the LV chamber of CAL mice, and after $6 \mathrm{~h}$, the heart and filter organs were collected. Percentage of migrated cells was calculated after performing real-time PCR for GFP. A mean of three independent experiments run in triplicate is shown ( $\left.{ }^{*} \alpha<0.02\right)$. (c) SDF-1-pretreated cardiac mesoangioblasts, transfected or not with vectors expressing L-selectin and GFP, were induced to migrate for $6 \mathrm{~h}$ through endothelium-coated filters and in the presence of mature cardiomyocytes in the lower chamber. A mean of three independent experiments run in duplicate is shown (left; ${ }^{*} \alpha<0.01$ ). A representative image of the transmigrated SDF-1-pretreated cardiac mesoangioblasts transfected with L-selectin is also shown (right). Bar, $30 \mu \mathrm{m}$. (d) SDF-1-pretreated cardiac mesoangioblasts, transfected or not with vectors expressing L-selectin-EGFP, as well as control cardiac mesoangioblasts were injected into the LV chamber of CAL mice, and after $6 \mathrm{~h}$, the heart and filter organs were collected and analyzed by real-time PCR for the presence of GFP. A mean of six independent experiments run in triplicate is shown $\left({ }^{*} \alpha<0.01\right)$

modified (SDF-1 plus L-selectin) cardiac mesoangioblasts into the LV chamber of C57 mice that underwent CAL. Mice were then killed 6 weeks after injection, and the heart (free wall) and filter organs (such as the liver, spleen and lungs) were harvested and RNA was extracted. The percentage of migrated and survived cardiac mesoangioblasts in each recipient organ (engraftment) was calculated by real-time PCR for GFP expression as a percentage of the value corresponding to the total of injected cells, normalized to their respective sham. As shown in Figure $5 \mathrm{a}, \sim 10 \%$ of controlinjected cells were found in the heart after 6 weeks, whereas $>35 \%$ of injected modified cardiac mesoangioblasts were found in the same region. Similar results were obtained when real-time PCR was performed for the $y$ chromosome as double control (Supplementary Figure S1c). To further verify these percentages, the GFP-positive population was sorted from digested hearts that had been injected 6 weeks before with control or treated cells. As shown in Figure $5 b$, the GFP-positive population was two times more abundant in the case of pretreated cells (SDF-1 plus L-selectin) than with control cells. As shown in Figure $5 \mathrm{c}$, control cardiac mesoangioblasts remained mainly in the ventricle wall (see arrowheads), whereas modified cardiac mesoangioblasts colonized larger areas inside the myocardium. Interestingly, these modified GFP-cardiac mesoangioblasts could also differentiate into new CMCs expressing sarcomeric actin 6 weeks after injection and therefore regenerate the damaged tissue (Figures 5d and e and Supplementary Figure S2). In fact, protein extraction from CAL-injected hearts confirmed the presence of injected GFP-cardiac mesoangioblasts inside the ventricle; it was found that the quantity of modified cardiac mesoangioblasts was higher after 6 weeks than the number of control mesoangioblasts (Figure $5 \mathrm{f}$ ). It is noteworthy that GFP-cardiac mesoangioblasts isolated from the free wall of the myocardium also expressed the sarcomeric actin protein 6 weeks after injection as analyzed by western blot (Figure $5 f$, lower panel), as well as late cardiac differentiation genes analyzed by real-time PCR (Figure 5g). Finally, before killing mice, we performed echocardiography studies that revealed a slight improvement in ventricular wall thickness, a two-fold increase of ejection and shortening fraction (SF), as well as minor changes in LV chamber diameter, 6 weeks after modified (SDF-1+ L-selectin) cardiac mesoangioblasts injection (Table 1).

Therefore, the combined treatment of cardiac mesoangioblasts with SDF-1 and L-selectin increases the quantity of cells that arrive to the damaged heart and improves the myocardium structure to a varying extent.

Human cardiac mesoangioblast migration to the heart is also susceptible of being regulated by SDF-1 pretreatment and L-selectin expression. To test whether a similar protocol would be also suitable for human cells, we used human cardiac mesoangioblasts that have been 
described before to reconstitute the myocardium after injection into SCID mice. ${ }^{15}$ They have morphology and gene expression similar to their mouse counterparts, a finite lifespan and the ability to differentiate into CMCs. Human cardiac mesoangioblasts were serum starved and then subjected to transmigration assays using Transwell chambers.

As shown in Figure 6a, human cardiac mesoangioblasts were also unable to cross endothelium-coated filters in the absence of stimuli. However, mature CMCs or pretreatment of
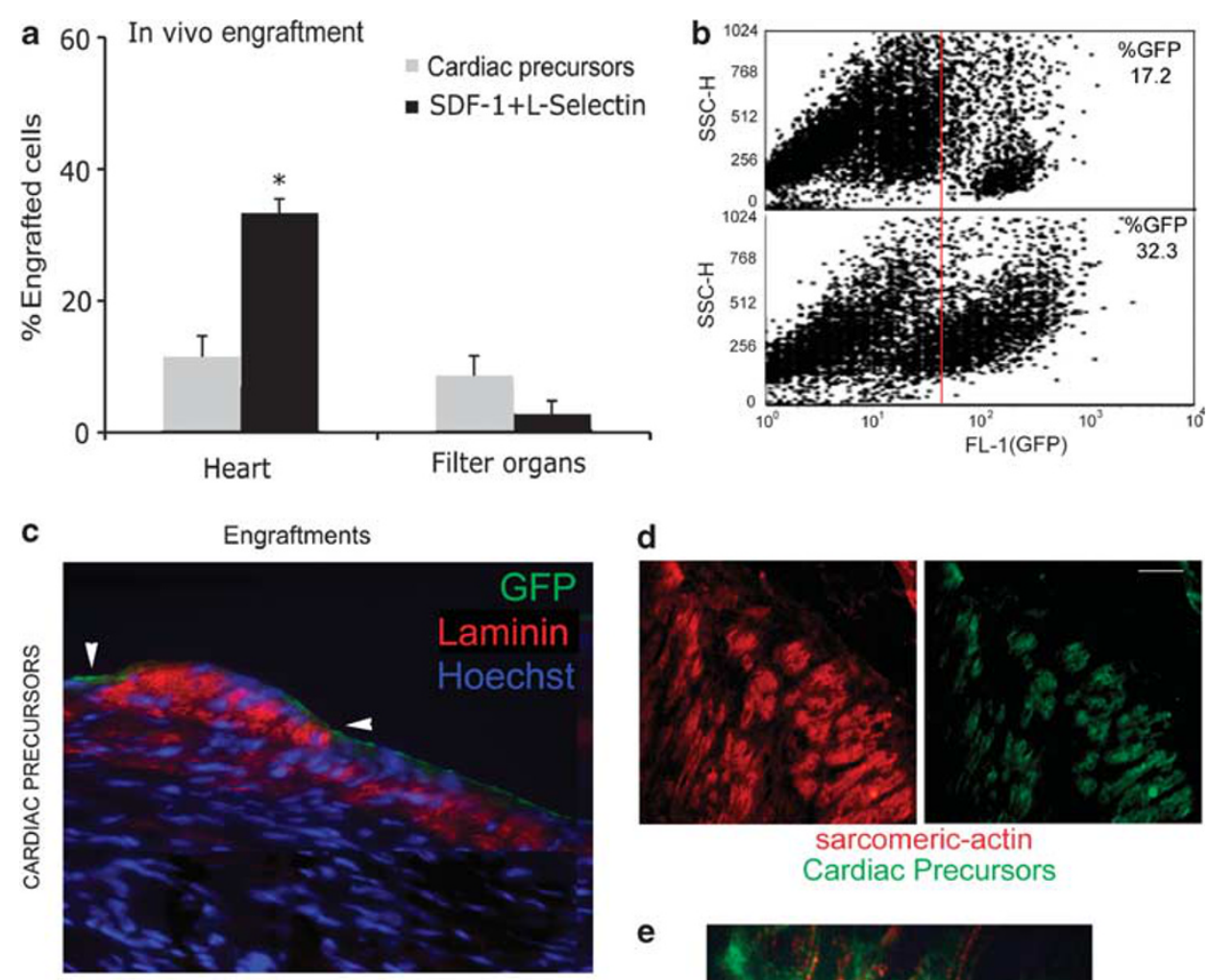

d
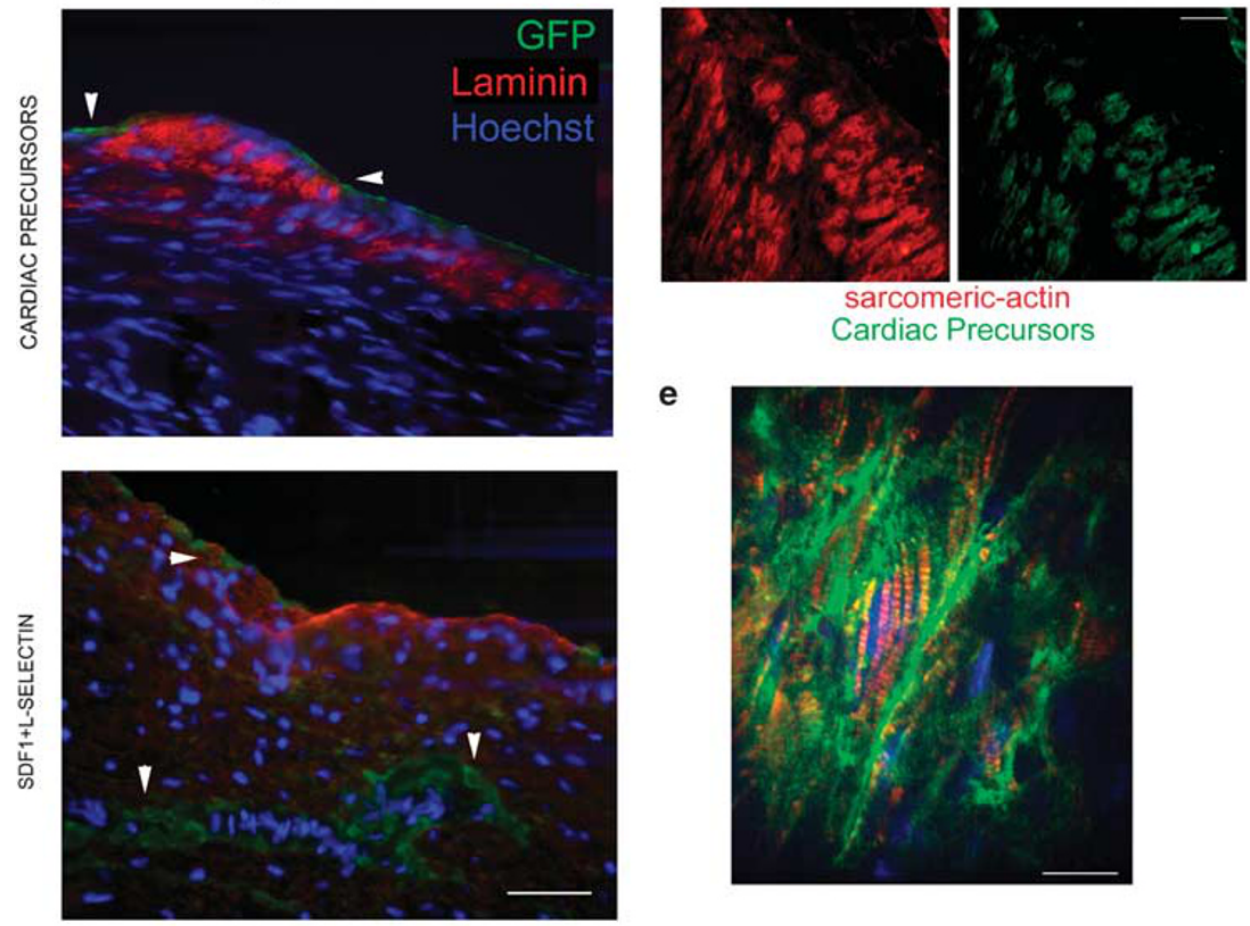

sarcomeric-actin

Cardiac Precursors

e

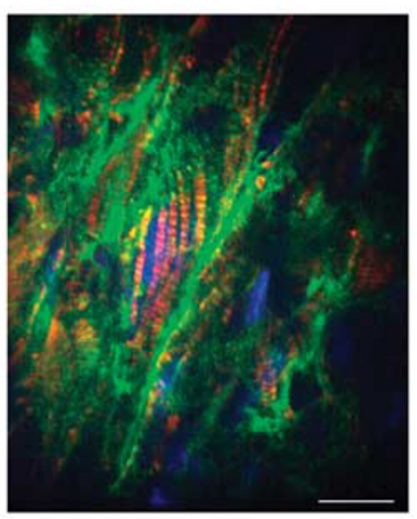

f
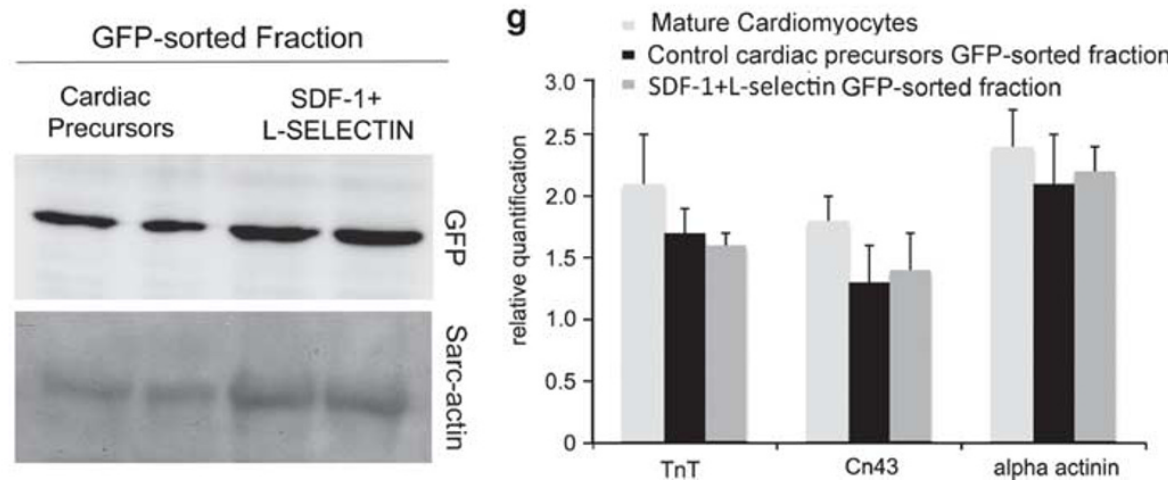
cells with SDF-1 induced from five- to eight-fold human cardiac mesoangioblast migration.

We then transfected human cardiac mesoangioblasts (that also do not express these molecules; Tagliafico $\mathrm{E}$ and Cossu $\mathrm{G}$, unpublished observations) with vectors expressing L-selectin and, after transfection, selected GFP-positive cells. As shown in Figure 6a (right column), SDF-1 pretreatment of L-selectin-expressing human cardiac mesoangioblasts increased threefold their ability to cross the endothelial barrier in Transwell assays. We then tested whether these in vitro results could be translated into an animal model for infarction. Therefore, human cardiac mesoangioblasts pretreated with SDF-1 and transfected with vectors encoding L-selectin were injected into the LV chamber of CAL-SCID mice that do not reject human cells. As shown in Figure 6b, almost $45 \%$ of human-modified (SDF-1-pretreated and L-selectintransfected) cardiac mesoangioblasts reached the infarcted free wall of the heart, $6 \mathrm{~h}$ after injection into the LV chamber, with a concomitant reduction in the number of cells detected in filter organs.

Taken together, these data show that it is possible to increase homing to the damaged area of the heart of human cardiac mesoangioblasts with an experimental protocol similar to that developed for mouse cardiac mesoangioblasts.

\section{Discussion}

Ischemic heart disease is today the major cause of cardiac failure, and thus is a leading cause of death worldwide. Cell therapy protocols, using different types of stem cells, have

Table 1 Ecocardiography values of the hearts coming from mice 6 weeks after injection of control or cardiac mesoangioblasts transfected with L-selectin and pretreated with SDF-1

\begin{tabular}{lrccc}
\hline & PBS & $\begin{array}{c}\text { SDF-1+ } \\
\text { Cardiac } \\
\text { precursors } \\
\text { c-sectin } \\
\text { cardiac } \\
\text { precursors } \\
\boldsymbol{n}=\mathbf{8}\end{array}$ & $\begin{array}{c}\text { P-value } \\
\text { Treated } \\
\text { cardiac }\end{array}$ \\
$\begin{array}{lrccc}\text { precursors } \\
\text { versus PBS }\end{array}$ \\
\hline LVIDd (mm) & 4.32 & 4.73 & 4.89 & 0.43 \\
EF (\%) & 27.12 & 34.28 & 41.17 & 0.032 \\
FS (\%) & 17.65 & 25.47 & 33.10 & 0.0204 \\
LVAWThd (mm) & 0.49 & 0.60 & 0.72 & 0.0415 \\
\hline
\end{tabular}

achieved partial success. ${ }^{16-21}$ Until now, intravascular or intra-myocardial delivery of stem cells to the heart seemed the most promising because of a partial but significant restoration of cardiac muscle structure and function. However, the migration and differentiation potency of stem cells, as well as their ability for regeneration are technical issues that have to be solved. In this work, we provide a new experimental strategy, based on pretreatment of donor cells with cytokines and surface expression of certain adhesion molecules, which improves homing of cardiac mesoangioblasts to the damaged heart.

Testing cardiac mesoangioblast-migratory activity in vitro. We initially measured cardiac mesoangioblast migration with a Transwell assay, already proven as a reliable indicator of the mechanisms that govern cellular trafficking in vivo. ${ }^{22}$ Cardiac mesoangioblasts cross the endothelium and migrate towards mature $\mathrm{CMCs}$, an in vitro setting mimicking the microenvironment. Likely, some factors secreted by these mature CMCs stimulate cardiac mesoangioblast migration as it has been demonstrated for other cell types. ${ }^{23-25}$ Microarray analysis had also shown that cardiac mesoangioblasts express several receptors for cytokines and therefore may respond to factors released by inflammatory cells or CMCs during myocardial infarction. Indeed, we found that SDF-1 was the most potent inducer of cardiac mesoangioblast migration, more than TNF- $\alpha$, previously described as a chemoattractant for skeletal muscle mesoangioblasts ${ }^{13}$ or for mesenchymal adipose stem cells. ${ }^{11}$ SDF-1 has previously been described to be present in the infarcted area. ${ }^{10}$

Cardiac mesoangioblasts homing to cardiac tissue in vivo. Homing to cardiac tissues in vivo proceeds through multiple mechanisms. When WT cardiac mesoangioblasts were injected into the LV chamber of the heart in infarcted or WT mice, only a small percentage of injected cells reached the injured myocardium, whereas most cells ended trapped in filter organs. Interestingly, the percentage of donor cells that could be recovered from the infarcted zone of the heart was significantly higher than that from the spared myocardium or from control hearts, possibly linked to the different cytokines pattern expressions in CMCs exposed to ischemic injury. ${ }^{26-28}$ In fact, when injected into

Figure 5 Pretreatment of mouse cardiac mesoangioblasts increases myocardium regeneration. (a) Infarcted hearts, injected with cardiac mesoangioblasts pretreated with SDF-1 and transfected with the L-selectin-EGFP vector or with control cardiac mesoangioblasts, were collected after 6 weeks and subjected to real-time PCR for the presence of GFP. A mean of seven independent experiments run in triplicate is shown ( $\left.{ }^{*} \alpha<0.02\right)$. (b) Infarcted hearts, injected with cardiac mesoangioblasts pretreated with SDF-1 and transfected with the L-selectin-EGFP vector or with control cardiac mesoangioblasts, were collected after 6 weeks and sorted for the GFP population after digestion. A representative plot of four independent experiments is shown. (c) Immunostaining of the free wall collected 6 weeks after injection with control cardiac mesoangioblasts or with cardiac mesoangioblasts pretreated with SDF-1 and transfected with the L-selectin-EGFP vector. Laminin is shown in red. Nuclei are stained with Hoechst (blue). Magnification: $\times 450$. Scale bar, $100 \mu \mathrm{m}$. (d) Immunofluorescence for GFP and sarcomeric actin (red) from a heart region of CAL mice 6 weeks after injection with cardiac mesoangioblasts pretreated with SDF-1 and transfected with L-selectin-EGFP. Scale bar, $100 \mu \mathrm{m}$. (e) Higher magnification immunofluorescence for GFP and sarcomeric actin (red) from the hearts of CAL mice 6 weeks after injection with cardiac mesoangioblasts pretreated with SDF-1 and transfected with L-selectin-EGFP. Nuclei are stained with Hoechst (blue). Scale bar, $20 \mu \mathrm{m}$. (f) Western blot for GFP and sarcomeric actin of total GFP-sorted fractions recovered from the hearts of CAL mice 6 weeks after injection with control GFP-cardiac mesoangioblasts or with cardiac mesoangioblasts pretreated with SDF-1 and transfected with the L-selectin-EGFP vector. One representative out of five independent experiments is shown. (g) Real-time PCR for late cardiac differentiation genes on mature cardiomyocytes and GFP-sorted fraction coming from the hearts of CAL mice 6 weeks after injection with cardiac mesoangioblasts, both control or pretreated with SDF-1 and transfected with the L-selectin-EGFP vector. Quantities have been normalized. A mean of four independent experiments run in triplicate is shown 
a
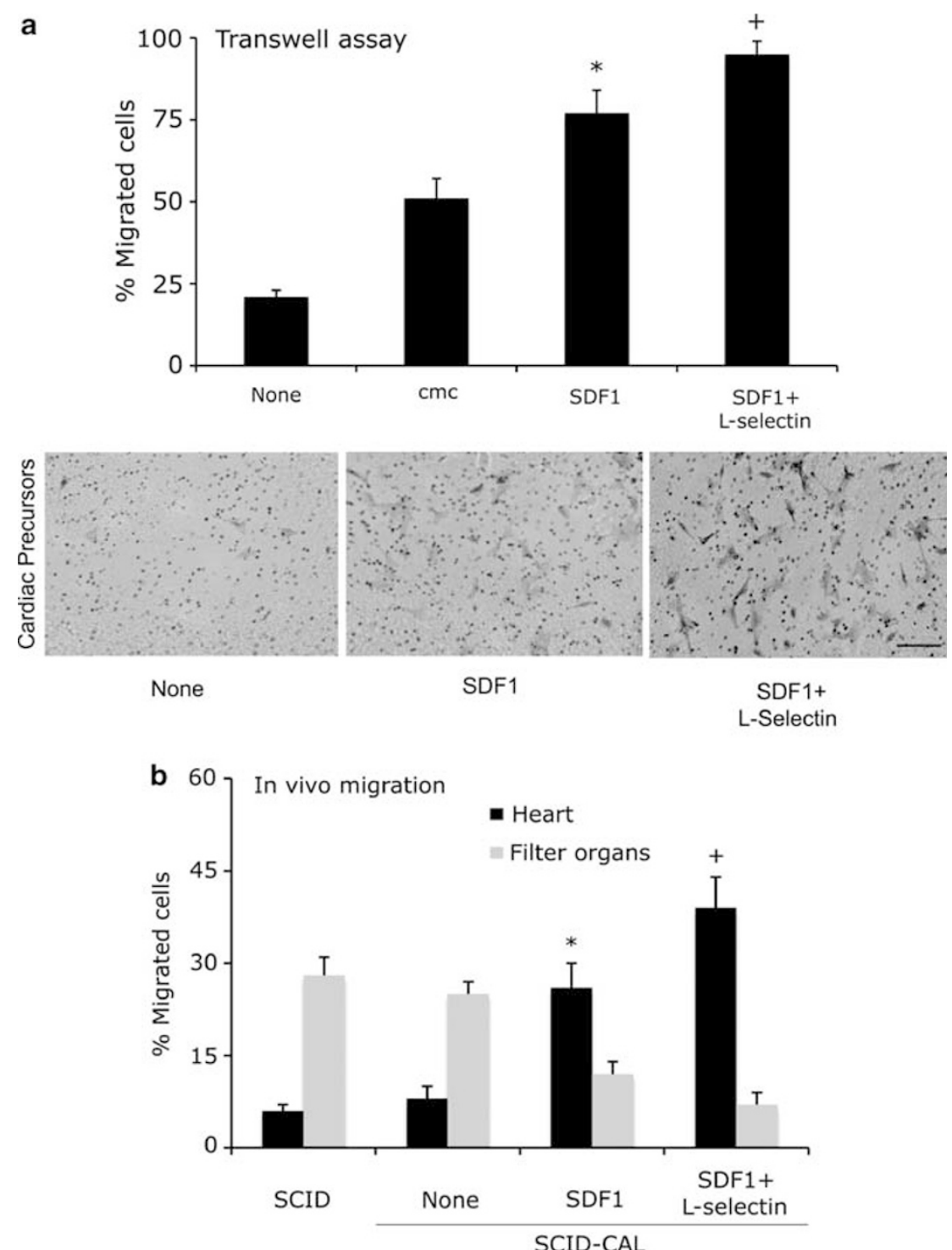

Figure 6 Pretreatment of human cardiac mesoangioblasts also improves homing to the heart. (a) Human cardiac mesoangioblasts pretreated with SDF-1 or transfected with L-selectin construction were plated on endothelium-coated filters and induced to migrate for $6 \mathrm{~h}$ in the presence of cardiomyocytes. One representative out of three independent experiments run in duplicate is shown. (left; ${ }^{*} \alpha<0.02,{ }^{+} \alpha<0.01$ ). A representative image of the transmigrated SDF-1-pretreated cardiac mesoangioblasts transfected or not with L-selectin is also shown (right). Bar, $30 \mu \mathrm{m}$. (b) Human cardiac mesoangioblasts transfected with vectors expressing L-selectin and GFP and pretreated with SDF-1 were injected into the LV chamber of CAL-SCID mice, and after $6 \mathrm{~h}$, the heart and filter organs were collected. The percentage of migrated cells is calculated after performing real-time PCR for GFP with the different samples. A mean of five independent experiments run in triplicate is shown $\left({ }^{*} \alpha<0.025,{ }^{+} \alpha<0.015\right)$

the LV chamber, cardiac mesoangioblasts accumulate in the first capillary filter they encounter and migrate outside the vessel, but only in the presence of inflammation, as in the case of infarcted heart. Indeed, cardiac mesoangioblasts express many receptors for inflammatory cytokines and migrate in vitro and in vivo in response to different stimuli.

Enhancing cardiac mesoangioblast-migratory activity. Previous work had shown that HMGB-1 or TNF- $\alpha$ stimulates proliferation and migration of skeletal muscle mesoangioblasts to dystrophic muscle. ${ }^{12,13}$ As in the case of skeletal muscle mesoangioblasts, we hypothesized that different stem cells would use different cytokine receptors for homing to the tissue of interest. In the case of cardiac mesoangioblasts, we observed that SDF-1 was the most potent molecule as chemoattractant. Interestingly, our work shows that pretreatment of donor cells with this cytokine is sufficient to increase expression of certain surface proteins necessary for migration and extravasation. We further investigated the mechanisms involved in this migration increment through a low-density array performed with cardiac mesoangioblasts pretreated with SDF-1. This experiment revealed that SDF-1 acts through CD44, a molecule that has been described to help in the rolling and extravasation of inflammatory cells. ${ }^{29,30}$ However, cardiac mesoangioblasts do not normally express many of these leukocytes molecules required for an efficient arrest at blood 
vessels, which may explain why most of the injected cells do not bind efficiently and are finally trapped inside filter organs. Therefore, L-selectin and $\beta 2$ integrin were expressed in cardiac mesoangioblasts to study their migration ability. The expression of L-selectin resulted in an eight-fold increase in cardiac mesoangioblast transmigration in vitro, and the number of cells that reached the damaged area was also doubled in vivo, reducing their number in filter organs. The combined strategies (i.e., pretreatment with SDF-1 of cardiac mesoangioblasts expressing L-selectin) resulted in a 15-fold increment of transmigration through the endothelium in vitro and 5-fold enhanced migration in vivo. Finally, we demonstrate that modified cardiac mesoangioblasts are not only able to reach the heart but also to colonize large areas of the myocardium where they can differentiate into mature CMCs and regenerate the damaged tissue.

In the perspective of cell therapy in patients, it is important to test whether a similar protocol will also work with human cells. Human cardiac mesoangioblasts have been isolated from fragments of vessels inside biopsies of the adult human heart and their characterization has been reported. ${ }^{15}$ Human cardiac mesoangioblasts were also exposed to SDF-1 and transfected with L-selectin and, under these conditions, reached cardiac tissues of infarcted-SCID mice three-fold more efficiently than did their corresponding untreated cells. Although these data suggest that further work may still be required to optimize human cardiac mesoangioblasts homing to the heart, they clearly indicate that the basic homing mechanisms are similar in mouse and humans and are equally susceptible to enhancement.

In conclusion, our results suggest that pretreatment of cardiac mesoangioblasts with SDF-1 and the presence of L-selectin are required for an efficient intra-vascular delivery of stem cells to the injured heart. Pretreatment of stem cells with cytokines is a simple procedure, and inclusion of a relatively small cDNA in a viral vector is also a feasible procedure in the context of autologous cell therapy with engineered stem cells. Taken together, these strategies represent a novel and simple method for improving the homing of stem cells to the damaged heart and for increasing the regeneration area and could be tailored in the future to different types of stem cells in clinical cell therapy protocols.

\section{Materials and Methods}

Reagents. Anti-GFP Ab was purchased from Chemicon (Temecula, CA, USA) Anti- $\alpha$ V integrins and anti-CD44 Abs were obtained from Serotec (Raleigh, NC, USA). Most reagents and some Abs were purchased from Sigma (St. Louis, MO, USA), unless stated otherwise. All the cytokines used were obtained from Peprotech Inc. (Rocky Hill, NJ, USA). GM1489, the MMP inhibitor, was obtained from Calbiochem (EMD Biosciences Inc., Darmstadt, Germany). Alexa-488 labeling kit was from Molecular Probes Inc. (Leiden, The Netherlands). Cav-1 siRNA was generated and used as described before. ${ }^{31}$

C57BL10 WT and SCID-beige mice were purchased from Charles River Laboratories (Wilmington, MA, USA) and Homo-EGFP mice were obtained from The Jackson Laboratory (Bar Harbor, ME, USA). All mice were handled following institutional guidelines.

Cell cultures. Neonatal CMCs were cultured in DMEM plus 20\% FBS and induced to differentiate in DMEM plus $2 \%$ FBS. Cardiac mesoangioblasts were isolated from control or Homo-EGFP male mice and from human ventricle explants, cloned and expanded as described previously. ${ }^{14,15}$ Constructs have been described previously ${ }^{13}$ and were transfected into mice or human cardiac mesoangioblasts with Lipofectamine (Invitrogen, Carlsbad, CA, USA), and positive clones were selected by sorting. Human cardiac mesoangioblasts transduced with a lentiviral vector encoding the GFP protein were used for some in vivo experiments. Murine microvascular endothelial H5V cells were grown in DMEM plus 10\% FBS and starved for $12 \mathrm{~h}$ before functional experiments.

Real-time PCR. Total RNA from different organs was isolated with TRIzol protocol (Invitrogen) and reverse transcribed using the Taqman kit (Platinum Taq DNA polymerase, Invitrogen). Real-time quantitative PCR was performed on an Mx3000P real-time PCR system (Stratagene, La Jolla, CA, USA). Each cDNA sample was amplified in duplicate using the SYBR Green Supermix (Bio-Rad, Hercules, CA, USA) for GFP or male y-chromosome detection as double control (primers GFP: forward, AAGTTCATCTGCACCACCG, reverse, TCCTTGAAGAAG ATGGTGCG; primers male y chromosome: forward, AAGCGACCCATGAACGCA TT, reverse, TTCGGGTATTTCTCTCTGTC). Primers for cardiac differentiation were used as described previously. ${ }^{14}$ Data are expressed as the percentage of migrated cells, which is calculated by comparing the concentration of target gene in our sample with the total input of injected cells.

Western blot. Tissues or GFP-sorted fractions were homogenized and proteins extracted and lysed directly in Laemmli buffer on ice. Lysates were resolved on $10 \%$ SDS-PAGE under reducing conditions, and proteins were transferred to nitrocellulose membrane (Hybond-ECL, Amersham Biosciences, Piscataway, NJ, USA). Membranes were revealed with anti-GFP, anti-sarcomeric actin and antitubulin Abs.

In vitro transwell migration assay. Transwell filters of 8-mm thickness (Corning Incorporated, Acton, MA, USA) were coated with 1\% gelatin, and endothelial H5V cells (previously pre-activated by $12 \mathrm{~h}$ exposure to TNF- $\alpha$ to induce the expression of surface antigens and adhesion molecules required for the appropriate extravasation) were plated to confluence on them for $24 \mathrm{~h}$. At the same time, neonatal CMCs were grown on a p24w plate with a differentiated medium for $0-4$ days. In all, $10^{4}$ mice cardiac mesoangioblasts, WT or pretreated (after a doseresponse assay (data not shown), the concentrations used were: $50 \mathrm{ng} / \mathrm{ml}$ and IL-8 SDF- $1 ; 80 \mathrm{ng} / \mathrm{ml}$ MCP-1; $30 \mathrm{ng} / \mathrm{ml} \mathrm{TNF}-\alpha ; 30 \mathrm{ng} / \mathrm{ml} \mathrm{FGF)} \mathrm{or} \mathrm{transfected,} \mathrm{were} \mathrm{plated}$ on the upper side of the Transwell chamber $24 \mathrm{~h}$ after plating $\mathrm{H} 5 \mathrm{~V}$ cells on the filter, and immediately thereafter, the chamber was then moved to wells containing differentiated CMCs to assay directed migration. After $6 \mathrm{~h}$ of transmigration, migrated cardiac mesoangioblasts were counted on the lower side of the filter. The results show migrated cells as a percentage of total input cells.

In vivo migration/grafting assay. WT, CAL mice aged 4 months were injected into the ventricle wall, ${ }^{32}$ with $5 \times 10^{5}$ GFP-cardiac mesoangioblasts. After 6,12 and $24 \mathrm{~h}$ or 6 weeks, animals were killed and different regions of the heart or filter organs were collected. RNA was extracted and a real-time PCR for GFP and male chromosome as double control (Supplementary Figure 1) was performed in all the samples as described above. Data are expressed as the percentage of migrated cells, which is calculated by comparing the concentration of target gene in our sample with the total input of injected cells.

The same samples were also processed for immunohistology against GFP; briefly, the heart was removed from mice previously injected with cardiac mesoangioblasts, and the LV free wall and the intraventricular septum and right ventricle were separated and frozen in liquid $\mathrm{N}_{2}$-cooled isopentane. Serial heart sections were fixed with 4\% PFA, permeabilized and immunostained as described previously (Galvez et al.) with anti-GFP, anti-laminin or anti-sarcomeric actin Abs. Alexa-488 or Alexa-594 (Molecular Probes, Eugene, OR, USA) were used as secondary staining $\mathrm{Ig}$ and DAPI for nuclear staining. Images were taken using a S100 TV microscope (Carl Zeiss Microlmaging Inc., Thornwood, NY, USA).

Echocardiography. Mice were anesthetized by inhalation of isoflurane/ oxygen $(1.25 \% / 98.75 \%)$ and examined by a $30 \mathrm{MHz}$ transthoracic echocardiography probe. Images were obtained with Vevo 770 (VisualSonics, Toronto, Canada) from mice 2 months after injection. Short-axis and long-axis, BMode and 2D M-mode views were recorded for off-line analysis. From these images, LV function was estimated by three validated methods as the SF, calculated as recommended by the American Society of Echocardiography ${ }^{33,34}$ and ejection fraction $(E F m)$ obtained from the $M$ mode, quantitative ejection fraction measured from the $B$ mode $(\mathrm{EFb})$ and visual assessment from the $B$ mode by an 
expert in echocardiography in a blind manner. Diastolic and systolic manual segmentations of the LV endocardial edge in four-chamber views of the heart were also measured. All data after analysis have been represented in a summary table (Table 1; LVIDd: LV diastolic diameter; EF: ejection fraction; FS: fractional shortening; LVAWThd: LV anterior wall diastolic thickness).

Prospective isolation. Control and injected hearts were finely minced and digested with collagenase/dispase (Sigma) digestion $\left(0.8 \mathrm{IU} / \mathrm{mg}\right.$ for $30 \mathrm{~min}$ at $37^{\circ} \mathrm{C}$ ). The cell suspension was filtered to remove tissue debris. Isolated cells were harvested and resuspended in $1 \mathrm{ml}$ of flow sort solution containing $5 \mu \mathrm{g} / \mathrm{ml}$ propidium iodide (Sigma) in PBS (Gibco Brl, Carlsbad, CA, USA). Cells were sorted for GFP (FL1) and PI (FL3) and collected for further experiments.

Microarray analysis. For mRNA analysis, total RNA was extracted from treated cardiac mesoangioblasts using TRIzol (Invitrogen Life Technologies, Carlsbad, CA, USA). CDNA was prepared by reverse transcription reaction and hybridized to the GEArray gene expression array MM-010 for extracellular matrix and adhesion molecules (SuperArray Bioscience Corporation, Frederick, MD, USA) according to the manufacturer's instruction. Data were subjected to densitometric analysis using GEArray Expression Analysis Suite (SuperArray Bioscience Corporation). RNA levels were expressed as the relative density after normalizing the hybridization signal to $\beta$-actin. The protein expression of positive candidate genes was confirmed by western blot (data not shown).

Cytokines measurements. Supernatants from fibroblast or CMC cultures were collected after 4 days of culture and analyzed using the mouse multi-cytokine detection system from Millipore (Billerica, MA, USA) for measuring cytokines according to the manufacturer's instructions. the Hearts from different animal models were homogenized in cell lysis buffer containing $0.1 \%$ igepal, and total protein concentration was determined using a DC protein assay kit and spectrophotometer (Bio-Rad). All heart samples were diluted with cell lysis buffer as required to a final total protein concentration of $500 \mathrm{~g} / \mathrm{ml}$ and cytokines were measured using the same kit protocol as for supernatants. Concentration of cytokines is represented in $\mathrm{pg} / \mathrm{ml}$ for supernatants values and in $\mathrm{pg} / \mathrm{mg}$ for tissue total protein values.

Statistical analysis. Statistical significance of the differences between the percentages values was assessed using the Kruskal-Wallis one-way ANOVA rank test. The $\alpha$-value represents significance.

\section{Conflict of Interest}

The authors declare no conflict of interest.

Acknowledgements. This study was supported by grants from the Spanish Ministry of Science and Innovation (SAF 2010-15239) to BGG, Heart Repair and Fondation Leducq to GC, and Ricerca Finalizzata bando 2007 from Ministerio della Salute to RL and GC. BGG acknowledges support from the 'Ramon y Cajal' tenure track programme from the Spanish Ministry of Science and Innovation (RYC200904669). The CNIC is supported by the Spanish Ministry of Science and Innovation and the Pro-CNIC Foundation.

1. Butcher E. Leukocyte-endothelial cell recognition: three (or more) steps to specificity and diversity. Cell 1991; 67: 1033-1036.

2. Fu S, Liesveld J. Mobilization of hematopoietic stem cells. Blood Rev 2000; 14: 205-218.

3. Grabovsky V, Feigelson S, Chen C, Bleijs D, Peled A, Cinamon G et al. Subsecond induction of alpha4 integrin clustering by immobilized chemokines stimulates leukocyte tethering and rolling on endothelial vascular cell adhesion molecule 1 under flow conditions. J Exp Med 2000; 192: 495-506.

4. Peled A, Kollet O, Ponomaryov T, Petit I, Franitza S, Grabovsky V et al. The chemokine SDF-1 activates the integrins LFA-1, VLA-4, and VLA-5 on immature human CD34(+) cells: role in transendothelial/stromal migration and engraftment of NOD/SCID mice. Blood 2000; 95: 3289-3296.

5. Springer T. Traffic signals for lymphocyte recirculation and leukocyte emigration: the multistep paradigm. Cell 1994; 76: 301-314.
6. Campbell J, Hedrick J, Zlotnik A, Siani M, Thompson D, Butcher E. Chemokines and the arrest of lymphocytes rolling under flow conditions. Science 1998; 279: 381-384.

7. Wright D, Bowman E, Wagers A, Butcher E, Weissman I. Hematopoietic stem cells are uniquely selective in their migratory response to chemokines. J Exp Med 2002; 195: $1145-1154$.

8. Peled A, Grabovsky V, Habler L, Arenzana-Seisdedos JS, Petit I, Ben-Hur H et al. The chemokine SDF-1 stimulates integrin-mediated arrest of CD34(+) cells on vascular endothelium under shear flow. J Clin Invest 1999; 104: 1199-1211.

9. Laterveer L, Lindley I, Heemskerk D, Camps J, Pauwels E, Willemze R et al. Rapid mobilization of hematopoietic progenitor cells in rhesus monkeys by a single intravenous injection of interleukin-8. Blood 1996; 87: 781-788.

10. Grunewald M, Avraham I, Dor Y, Bachar-Lustig E, Itin A, Jung S et al. VEGF-induced adult neovascularization: recruitment, retention, and role of accesory cells. Cell 2006; 124 175-189.

11. Galvez BG, San Martin N, Rodriguez C. TNF-alpha is required for the attraction of mesenchymal precursors to white adipose tissue in Ob/Ob mice. Plos One 2009; 4: e4444.

12. Palumbo R, Sampaolesi M, Marchis FD, Tonlorenzi R, Colombetti S, Mondino A et al. Extracellular HMGB1, a signal of tissue damage, induces mesoangioblast migration and proliferation. J Cell Biol 2004; 164: 441-449.

13. Galvez BG, Sampaolesi M, Brunelli S, Covarello D, Gavina M, Rossi B et al. Complete repair of dystrophic skeletal muscle by mesoangioblasts with enhanced migration ability J Cell Biol 2006; 174: 231-243.

14. Galvez BG, Sampaolesi M, Barbuti A, Crespi A, Covarello D, Brunelli S et al. Cardiac mesoangioblasts are committed, self-renewable progenitors, associated with small vessels of juvenile mouse ventricle. Cell Death Differ 2008; 15: 1417-1428.

15. Galvez BG, Covarello D, Tolorenzi R, Brunelli S, Dellavalle A, Crippa S et al. Human cardiac mesoangioblasts isolated from hypertrophic cardiomyopathies are greatly reduced in proliferation and differentiation potency. Cardiovasc Res 2009; 83: 707-716.

16. Beltrami A, Barlucchi L, Torella D, Baker M, Limana F, Chimenti $S$ et al. Adult cardiac stem cells are multipotent and support myocardial regeneration. Cell 2003; 114: 763-776.

17. Caplice N, Deb A. Myocardial-cell replacement: the science, the clinic and the future. Nature 2004; 1: 90-95.

18. Dawn B, Stein A, Urbanek K, Rota M, Whang B, Rastaldo R et al. Cardiac stem cells delivered intravascularly traverse the vessel barrier, regenerate infarcted myocardium, and improve cardiac function. Proc Natl Acad Sci USA 2005; 102: 3766-3771.

19. Linke A, Muller P, Nurzynska D, Casarsa C, Torella D, Nascimbene A et al. Stem cells in the dog heart are self-renewing, clonogenic, and multipotent and regenerate infarcted myocardium, improving cardiac function. Proc Natl Acad Sci USA 2005; 102: 8966-8971.

20. Oh H, Bradfute S, Gallardo T, Nakamura T, Gaussin V, Mishina $Y$ et al. Cardiac progenitor cells from adult myocardium: homing, differentiation, and fusion after infarction. Proc Natl Acad Sci USA 2003; 100: 12313-12318.

21. Urbanek K, Torella D, Sheikh F, Angelis AD, Nurzynska D, Silvestri F et al. Myocardial regeneration by activation of multipotent cardiac stem cells in ischemic heart failure. Proc Natl Acad Sci USA 2005; 102: 8692-8697.

22. Aiuti A, Webb I, Bleul C, Springer T, Gutierrez-Ramos J. The chemokine SDF-1 is a chemoattractant for human CD34+ hematopoietic progenitor cells and provides a new mechanism to explain the mobilization of $\mathrm{CD} 34+$ progenitors to peripheral blood. $J$ Exp Med 1997; 185: 111-120.

23. Abbott J, Huang Y, Liu D, Hickey R, Krause D, Giordano F. Stromal cell-derived factor-1 alpha plays a critical role in stem cell recruitment to the heart after myocardial infarction but is not sufficient to induce homing in the absence of injury. Circulation 2004; 110: 3300-3305.

24. Chavakis E, Aicher A, Heeschen C, Sasaki K, Kaiser R, El Makhfi N et al. Role of beta2integrins for homing and neovascularization capacity of endothelial progenitors cells. J Exp Med 2005; 201: 63-72

25. Kocher AA, Schuster MD, Bonaros N, Lietz K, Xiang G, Martens TP et al. Myocardial homing and neovascularization by human bone marrow angioblasts is regulated by IL-8/ Gro CXC chemokines. J Mol Cell Cardiol 2006; 40: 455-464.

26. Chang KH, Chan-Ling T, McFarland EL, Afzal A, Pan H, Baxter LC et al. IGF binding protein-3 regulates hematopoietic stem cell and endothelial precursor cell function during vascular development. Proc Natl Acad Sci USA 2007; 104: 10595-10600.

27. Chavakis E, Hain A, Vinci M, Carmona G, Bianchi ME, Vajkoczy $P$ et al. High-mobility group box 1 activates integrin-dependent homing of endothelial progenitor cells. Circ Res 2007; 100: 104-112

28. Jojo K, Li M, DW L. Endothelial progenitor cells in neovascularization of infarcted myocardium. J Mol Cell Cardiol 2008; 45: 530-544.

29. Mylona E, Jones KA, Mills ST, GK P. CD44 regulates myoblast migration and differentiation. J Cell Physiol 2006; 209: 314-321.

30. Zöller M, Gupta P, Marhaba R, Vitacolonna M, P F-P. Anti-CD44-mediated blockade of leukocyte migration in skin-associtated immune diseases. J Leuk Biol 2007; 82: 57-71.

31. Galvez BG, Matias-Roman S, Yañez-Mo M, Vicente-Manzanares M, Sanchez-Madrid F, Arroyo AG. Caveolae are a novel pathway for membrane-type 1 matrix metalloproteinase traffic in human endothelial cells. Mol Biol Cell 2004; 15: 678-687. 
32. Galli D, Innocenzi A, Staszewsky L, Zanetta L, Sampaolesi M, Bai A et al. Mesoangioblasts, vessel-associated multipotent stem cells, repair the infarcted heart by multiple cellular mechanisms. Arterioscler Thromb Vasc Biol 2005; 25: 692-697.

33. Sahn DJ, De Maria A, Kisslo J, Weyman A. Recommendations regarding quantitation in M-mode echocardiography; results of a survey of echocardiographic measurements. Circulation 1978; 58: 1072-1083.

34. Schiller NB, Shah PM, Crawford M, De Maria A, Devereux R, Feigenbaum $\mathrm{H}$ et al Recommendations for quantitation of the left ventricle by two-dimensional echocardiography American Society of Echocardiography Committee on Standards, subcommittee on Quantitation of Two Dimensional Echocardiograms. J Am Soc Echocardiogr 1989; 2: 358-367.

(c) This work is licensed under the Creative Commons Attribution-NonCommercial-No Derivative Works 3.0

Unported License. To view a copy of this license, visit http:// creativecommons.org/licenses/by-nc-nd/3.0

Supplementary Information accompanies the paper on Cell Death and Differentiation website (http://www.nature.com/cdd) 